\title{
Facilitating Developer-User Interactions with Mobile App Review Digests
}

Jeungmin Oh

Department of Knowledge

Service Engineering,

KAIST, Daejeon, Korea

jminoh@kaist.ac.kr

Daehoon Kim

Department of Knowledge

Service Engineering,

KAIST, Daejeon, Korea

daehoonkim@kaist.ac.kr

Uichin Lee

Department of Knowledge

Service Engineering,

KAIST, Daejeon, Korea

uclee@kaist.ac.kr

\section{Jae-Gil Lee}

Department of Knowledge

Service Engineering,

KAIST, Daejeon, Korea

jaegil@kaist.ac.kr

\section{Junehwa Song}

Department of Computer

Science,

KAIST, Daejeon, Korea

junesong@kaist.ac.kr

\author{
Abstract \\ As users are interacting with a large of mobile apps \\ under various usage contexts, user involvements in an \\ app design process has become a critical issue. Despite \\ this fact, existing apps or app store platforms only \\ provide a limited form of user involvements such as \\ posting app reviews and sending email reports. While \\ building a unified platform for facilitating user \\ involvements with various apps is our ultimate goal, we \\ present our preliminary work on handling developers' \\ information overload attributed to a large number of \\ app comments. To address this issue, we first perform \\ a simple content analysis on app reviews from the \\ developer's standpoint. We then propose an algorithm \\ that automatically identifies informative reviews \\ reflecting user involvements. The preliminary \\ evaluation results document the efficiency of our \\ algorithm.
}

\section{Author Keywords}

Mobile apps; app review; comment classification; user involvements; user-centered design

\section{ACM Classification Keywords}

I.5.2 [Design Methodology]: Classifier design and evaluation; H.1.2 [User/Machine Systems]: Human factors 


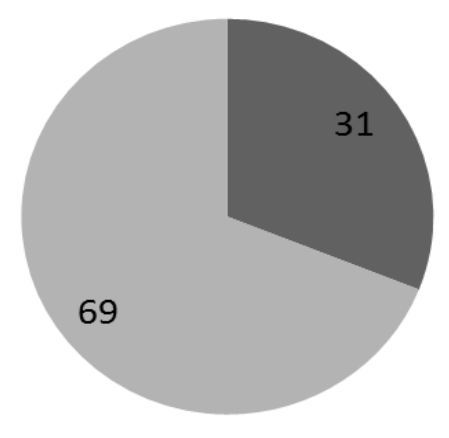

active Action $\quad$ Passive Action

Figure 1. "How do you react when you want to communicate with app designer regarding a mobile app?"

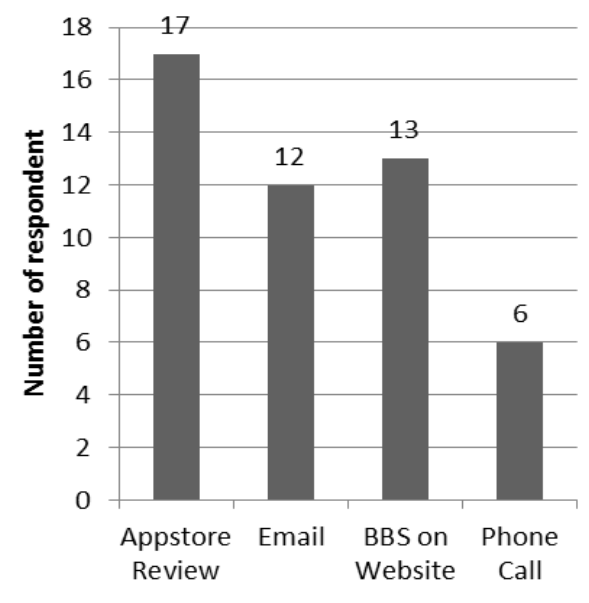

Figure 2. "Which methods do you use to communicate with developer?"

\section{Introduction}

User involvements in software design have been one of the important issues in software engineering $[1,2]$.

Understanding user needs and usage contexts would be the key factors in increasing the potential utility/value to the end users. For this reason, practitioners often perform various forms of user involvements across the development lifecycle including customer interviews, usability testing, beta deployment, and end user support

However, the widespread use of smartphone apps has significantly changed the traditional software development environment. App design is mostly utilitydriven, and rapid prototyping with a limited form of user involvements is often performed. Further, usage patterns of mobile apps are quite unique as opposed to existing software (e.g., short session time of mobile apps); and usage context also varies widely (e.g., at home, on the move). In this environment, we think that facilitating user involvements will help the developers to better understand user needs and usage context.

Existing apps and app store platforms often rely on a passive form of user involvement (e.g., posting app reviews in app stores or sending emails to the developers). In the case of open source apps, sometimes a bug tracking system is used to promote participation of grassroots developers (e.g., Bugzilla for Firefox for Andriod). This means that user participation is mostly passive (i.e., unilateral communication) and fragmented (across different app stores and locales). Likewise, developers are challenged with a large volume of app comment streams (from different app stores using heterogeneous devices/OSs, and with possibly different languages).

While building a unified platform for facilitating user involvements with various apps is our ultimate goal, as a first step, we focus on mitigating developers' information overload attributed to a large number of app comments. In the field of software engineering, there have been several studies on summarizing bug reports in often source software (e.g., Debian) [3, 4], but our work differs as we consider end-user feedback posted in app stores. In this paper, we investigate how developers and users interact within an app store environment. We present a method of filtering mobile app reviews to reduce the information overload of developers.

\section{Developer-User Interaction}

To get a basic understanding of how developers and users interact, we conducted a survey $(n=100)$ on user's motivation and behavior. The survey was administered to randomly chosen smartphone users via a survey research company. The majority of participants are in their 20 s (55\%), and $22 \%$ are under 20. $18 \%, 2 \%, 2 \%$ and $1 \%$ are in their 30 s, 40 s, 50 s and 60 s respectively. $40 \%$ are males.

Q1 (common) "How do you react when you want to communicate with app designer regarding a mobile app?"

Figure 1 shows that users are more likely to take a passive action such as deleting apps rather than to perform active actions such as writing an app store review or sending an email to the developer. To find the reason for this behavior, we asked an additional question both for active and passive groups separately. 
Q2 (active group) "Which methods do you use to communicate with developers?" (multiple answer) In addition to four original selections, which we derived by interviewing graduate students in the authors' department, we allow participants to report any other channels in a free-text format, but we didn't find any other channels. As shown in Figure 2, the result reveals that the most popular channel is writing app store reviews as we expected, but the traditional methods like phone calls or BBS are used.

Q3 (passive group) "What are your reasons for reacting passively?" (multiple answer)

Like the previous question, we gave optional openended text field to find out unknown reason of passiveness and we identified 'tiresome' from comments of four users. As shown in Figure 3, most users expected the inquiry would take long time to be responded or receive no response. It also indicates that a non-negligible portion of recipients were not even aware of which channel to use.

To summarize, users would like to communicate with developers using app store reviews. Additionally, the reasons why the user passively reacts were mainly due to low responsiveness.

\section{Filter for Classifying App Store Review}

Since per app review in app stores (e.g., Google Play, Apple App Store) is not mainly designed for reporting bugs as in bug tracking systems, it contains not only informative feedback but also simple expressions like a user's sentiment. Due to a large volume of app comments, developers spend a significant amount of time on checking reviews without any meaningful issue, for example "What an awesome app, comes in handy

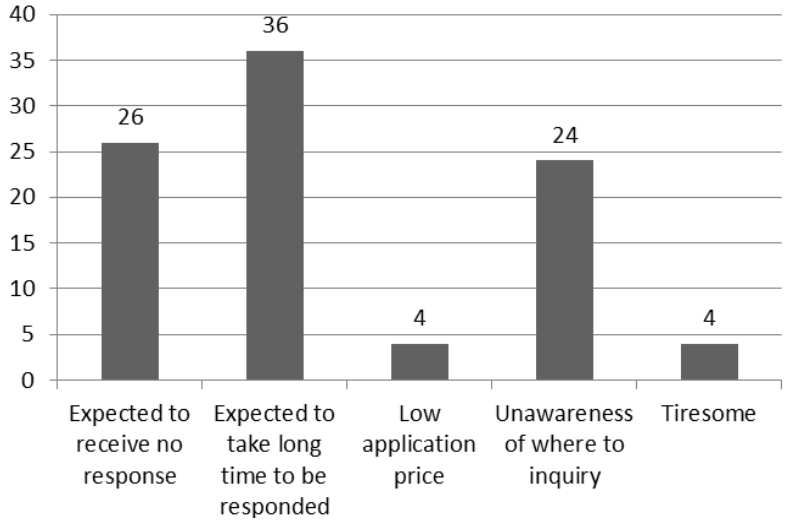

Figure 3. "What are your reasons for reacting passively?"

so many times a day. thank you", which is just mere expression of satisfaction. In this sense, a filter for classifying reviews which are less informative for developers could play an important role to save developer's time and energy, which otherwise could be used for quality/timely feedback.

To build a model that filters out uninformative comments, we used the following steps: (1) automated crawling publicly accessible reviews, (2) manual coding sampled reviews to decide whether each review contains issues from the developer's standpoint, (3) model construction/training/evaluation using the annotated dataset.

\section{Data Collection}

We collected public reviews of 24,000 applications in Google Play. Since Google Play doesn't provide the entire list of apps in search result, we put two digit combinations of alphanumeric characters as a search 


\begin{tabular}{|c|c|}
\hline Category & Description \\
\hline $\begin{array}{c}\text { Functional } \\
\text { Bug }\end{array}$ & $\begin{array}{l}\text { Functional bug of an } \\
\text { app; e.g., "Black } \\
\text { screen issue on the } \\
\text { Galaxy S3" }\end{array}$ \\
\hline $\begin{array}{l}\text { Functional } \\
\text { Demand }\end{array}$ & $\begin{array}{l}\text { Functional demands } \\
\text { about the features that } \\
\text { a user wishes to have. } \\
\text { e.g., "This seriously } \\
\text { needs a postcode } \\
\text { search! It's a must for } \\
\text { any navigation app. } \\
\text { Apart from that seems } \\
\text { to work fine" }\end{array}$ \\
\hline $\begin{array}{c}\text { Non- } \\
\text { functional } \\
\text { Request }\end{array}$ & $\begin{array}{l}\text { Non-functional request } \\
\text { or complaint such as } \\
\text { design and content } \\
\text { related issues: e.g., } \\
\text { "We want another car } \\
\text { and more stages and } \\
\text { more gas in high way } \\
\text { map." }\end{array}$ \\
\hline
\end{tabular}

query for building a list of apps. We crawled the reviews from November 21 to 28, 2012, and the total number of reviews is $1,711,556$. Each review contains an app's name, category, rating (in 5-levels), posting date, device, title and text.

\section{Manual Coding by Developers}

We performed preliminary content analysis and derived three issue categories as shown in Table 1. The categorization was done thoroughly in developers' standpoint because the readers of comments include people who are directly involved with software design and production. The issues consist of three categories as described in Table 1. We then classified 2,800 reviews into these issue categories and analyzed it in terms of rating and word counts to attain some insights into the patterns of app reviews. This data set was randomly chosen to avoid bias toward specific applications as follows: 20 reviews from 10 applications in 14 categories. The categories are determined by the raters who have mobile app programming experiences more than 1 year.

The coding results are presented in Figure 4. The reason why the sum of occurrences is not the same as the original number is that we excluded non-English and unreadable comments. The results reveal that $66 \%$ of reviews (1851 out of 2785) are uninformative for developers. The inter-rater agreement with Cohen's Kappa coefficient measured as 0.9025 indicating good agreement.

We also plotted the number of reviews in terms of word counts in Figure 6. It shows that there is a significant gap between the frequency of the overall comments

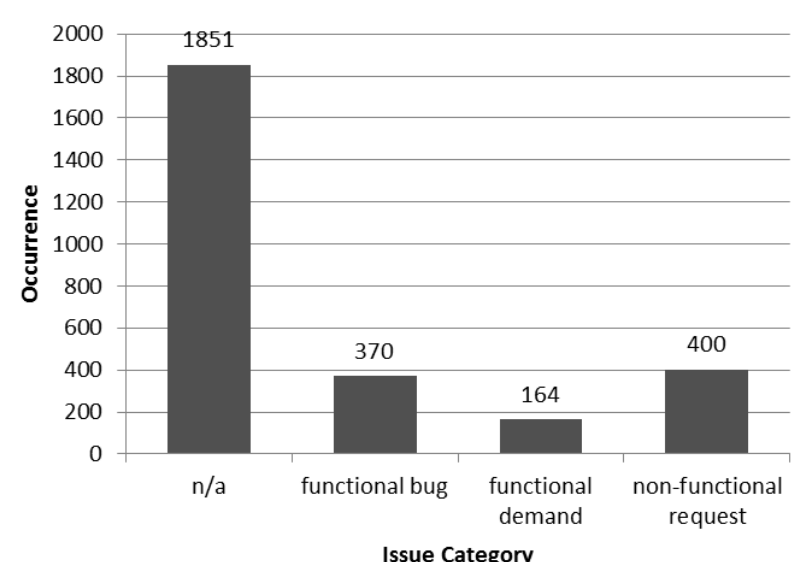

Issue Category

Figure 4. Distribution of categories including review without issue (marked as ' $n / a$ ')

and informative comments with respect to the word counts.

Issue Keyword Count

To find a set of keywords related to informative comments, we firstly ran Latent Dirichlet Allocation (LDA) on informative comments. However the result was unrecognizable even though it was examined by two developers. This means that existing automatic topic classifications like LDA cannot be directly used for finding informative comments. The reason why it fails is that the length of a comment is too short for producing topic keywords (mean=18.27 words)

Instead we devised a new method of extracting the keyword set implying informative comments. As a first step, we preprocessed the comment data using Natural Language ToolKit (NLTK) [5]: tokenize the word and apply stemming in order to clean the word set from 


\section{Set $_{\text {Issue-Only }}$ of our data set}

['update', 'text', 'rest', 'through', 'clos', 'nam', 'slow', 'const',

'tablet', 'delet', 'brows', 'send', 'wer', 'going', 'black', '4', 'photo', 'hap', 'els', 'hat', 'ic', 'crash', 'big', 'watch', 'disappoint', 'bit', 'thes', 'restart', 'mod', 'wont', 'bar', 'bas', 'level', 'button', 'each', 'whil', 'droid', 'tel', 'back', 'everytim', 'horr', 'fail', 'wo', 'click', 'er', 'freez', 'review', 'landscap', 'xper', '7', 'rec', 'pls', 'mem', 'screen', 'plz', 'reason', 'otherw', 'phone', 'wtf', 'fiv', 'post', 'wait', 'last', 'turn', 'menu', 'iphon', 'confus', 'col', 'pop', 'sometim', 'doesnt', 'miss', 'select', 'smal', 'crap', 'log', 'support', 'upgrad', 'long', 'next', 'avail', 'anym', 'interfac', 'until', 'buy', 'forc', 'screen.', 'uninstal', 'bug', 'annoy', 'account', 'hour', 'default', 'remov', 'un', 'ui', 'ful', 'control', 'almost', 'lock', 'sad', 'cant', 'rid', 'seem', 'again', 'end', 'min', 'receiv', 'siz', 'check', 'ent', 'useless', 'pag', 'instead', 'complet', 'poor', 'map', 'though', 'mess', 'lag', 'paid', 'alert', 'wrong', 'kil', 'why', 'icon', 'mobl', 'alarm', 'caus', 'pay', 'latest']

$* k=0.6$, bold words are strongly issuerelated words by two raters

\begin{tabular}{|c|c|c|c|}
\hline Features & Precision & Recall & F-measure \\
\hline Random & 0.3512 & 0.4790 & 0.4053 \\
\hline F1 & 0.8624 & 0.7306 & 0.7910 \\
\hline F2 & 0.6506 & 0.4984 & 0.5644 \\
\hline F3 & 0.8698 & 0.7033 & 0.7778 \\
\hline F1+F2 & 0.8591 & 0.7795 & 0.8174 \\
\hline F1+F3 & 0.8672 & 0.8224 & 0.8442 \\
\hline F1+F2+F3 & 0.8981 & 0.8165 & 0.8553 \\
\hline
\end{tabular}

Table 1. Result of classification (F1 : rating / F2 : word count

/ F3 : issue keyword count)

\section{Conclusion and Future Work}

We studied the role of user involvements in mobile app development by studying app comments in an app store. Our survey results of smartphone users show that the most popular interaction channel with smartphone users is app review sections in the app store, and thus, end users are fairly passive in terms of user involvements during the app development lifecycle. We also analyzed the content of app comments and showed that there are mainly three comment types from the developers' standpoint: functional bugs, and functional demands, and non-functional requests. Further, to lower the information overload due to a large volume of app review streams, we proposed a simple approach of automatically identify informative comments. Our preliminary analysis results show that the method achieves fairly high precision and recall.
In the future, we plan to propose and implement a unified software architecture that can facilitate developer-user interactions. We will also improve the performance of our model by using better classification algorithms, and adding more functions (e.g., topic classification). Further, a longitudinal field study of a user-centered design process is needed to better understand the importance of user involvements for mobile apps.

\section{Acknowledgements}

This research was supported by the SW Computing R\&D Program of KEIT (2012,10041313, UX-oriented Mobile SW Platform) funded by the Ministry of Knowledge Economy.

\section{References}

1] Michael Gallivan. The user-developer

communication process: a critical case study. Blackwell Publishing Ltd (2003).

[2] Mark Keil, Erran Carmel. Customer-Developer Links in Software Development. Communication of the ACM (1995).

[3] Sarah Rastkar, Gail C. Murphy. Summarizing software artifacts: a case study of bug reports. ACM/IEEE International Conference on Software Engineering (ICSE 2010).

[4] Lotufo, R., Z. Malik, and K. Czarnecki. Modelling the 'Hurried' Bug Report Reading Process to Summarize Bug Reports. Proceedings of the International Conference on Software Maintenance (ICSM 2012).

[5] Natural Language ToolKit (NLTK). http://nltk.org.

[6] C.-C. Chang and C.-J. Lin. LIBSVM : a library for support vector machines, ACM Transactions on Intelligent Systems and Technology, 2:27:1--27:27, 2011. 